\title{
An effective routing with delay minimization for multi-hop wireless mesh network
}

Document Version

Accepted author manuscript

Link to publication record in Manchester Research Explorer

\section{Citation for published version (APA):}

Chai, Y., \& Zeng, X. (Accepted/In press). An effective routing with delay minimization for multi-hop wireless mesh network. In IEEE Global Communications Conference

\section{Published in:}

IEEE Global Communications Conference

\section{Citing this paper}

Please note that where the full-text provided on Manchester Research Explorer is the Author Accepted Manuscript or Proof version this may differ from the final Published version. If citing, it is advised that you check and use the publisher's definitive version.

\section{General rights}

Copyright and moral rights for the publications made accessible in the Research Explorer are retained by the authors and/or other copyright owners and it is a condition of accessing publications that users recognise and abide by the legal requirements associated with these rights.

\section{Takedown policy}

If you believe that this document breaches copyright please refer to the University of Manchester's Takedown Procedures [http://man.ac.uk/04Y6Bo] or contact uml.scholarlycommunications@manchester.ac.uk providing relevant details, so we can investigate your claim.

\section{OPEN ACCESS}




\title{
An effective routing with delay minimization for multi-hop wireless mesh network
}

\author{
Yuan Chai, Xiao-Jun Zeng \\ School of Computer Science, the University of Manchester, Manchester, UK, M13 9PL \\ Email: yuan.chai-2@postgrad.manchester.ac.uk, x.zeng@manchester.ac.uk
}

\begin{abstract}
As wireless communication develops rapidly, nextgeneration networks have higher demand for short delay. Routing is an important part of network configuration, and can influence the network performance including delay significantly. Thus designing an effective routing is essential. To achieve the global best network performance, the problem of routing can be modeled as a mathematical optimization problem. An effective routing with the objective of delay minimization for multi-hop wireless mesh network (RODM) is proposed in this paper. Delay is derived according to interference, bandwidth and probability of transmission failure. As the link between two neighboring nodes is bidirectional, RODM considers the asymmetrical transmission failure probabilities of two-direction links between two neighbor nodes. The relationship between delay and the number of interfering nodes is then built. To solve this optimization problem, an improved genetic algorithm is proposed to balance load. The dynamic network performance produced by different chosen routes is also considered. Simulation results show that RODM can obtain better network performance.
\end{abstract}

\section{INTRODUCTION}

Wireless mesh network (WMN) is low-cost, selfconfiguration and self-healing, which has a wide range of applications such as military communication, internet of things, and emergency communication [1] [2]. The multihop feature can help WMN extend its flexible coverage area. The network model of WMN is shown in Fig. 1.

WMN contains mesh routers and mesh clients [3]. The mesh router with stronger function and wired interface to the Internet can be seen as a gateway. Mesh routers can have multiple radio interfaces banded with orthogonal wireless channels. Mesh clients always have only one radio interface. Destinations of data traffic in WMN can be gateway or

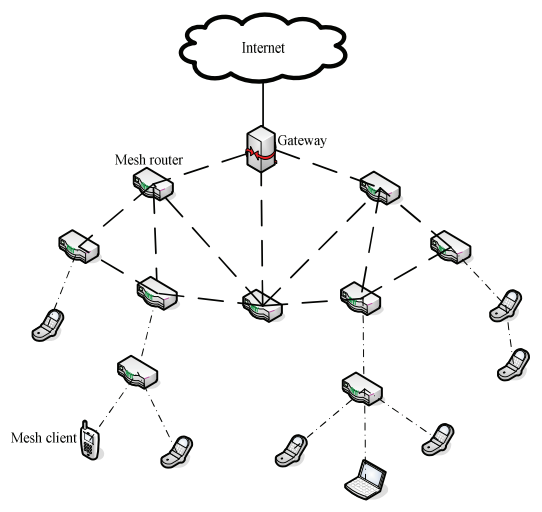

Fig. 1. Network model of WMN mesh nodes [4]. In both traffic types, data packets can be forwarded by multiple hops. Routing is to select effective paths from sources to destinations by considering the condition of multiple mesh nodes on the whole path. To obtain global best network performance, routing can be modeled as an optimization problem [5]. As more and more users require the service with little delay nowadays, such as video and autonomous cars, delay is a very essential factor that should be considered to be minimized when selecting paths. The Routing with the Objective of Delay Minimization for wireless mesh network (RODM) proposed in this paper builds an optimization model with constraints. The main contributions of RODM can be summarized as follows:

- Build an optimization model to solve routing problem in multi-hop WMN. For the objective of minimizing delay, the mathematical expression of delay is derived. In the process of modeling delay, bandwidth, interference, Signal to Interference plus Noise Ratio (SINR), transmission failure probability and asymmetry of link are all considered. The total transmission failure probability of each hop can be obtained by the packet loss probabilities of forward and reverse links, and this probability is influenced by interference. The relationship between delay and the number of interfering nodes is built in first time. Delay can then be easily expressed without proactive detecting.

- Improve the genetic algorithm (GA) [6] to solve the optimization problem. According to the derived relationship between delay and number of interfering mesh nodes, the improved GA can select a replaced node with least number of interfering nodes to avoid multiple data flows using a same congested node. The dynamic network condition caused by route selection is also considered. RODM checks the number of interfering nodes every time of finding optimal paths in the iterative optimization.

The structure of this paper is as follows. Section II reviews some related research. Section III explains RODM in detail. Evaluation results are given in Section IV, and Section $\mathrm{V}$ gives the conclusion.

\section{RELATED WORKS}

The researches about routing optimization problem have two focuses: the model establishment and the improvement of solution method. For the part of model establishment, one search-based routing is proposed in [7]. It considers 
expected transmission time to evaluate link quality, and uses GA to solve the optimization problem. The method in [8] gives a method of evaluating end-to-end throughput, which can guide routing. It considers the relationship among neighboring interfaces and transmission rate. IQoSR [9] considers the constraints of delay, throughput and packet error ratio. The weight values of these parameters will be different according to different quality of service (QoS) flow types. The routing proposed in [10] builds a mixed integer linear program model by maximizing throughput. Different interference relationships are considered when calculating link capacity. LARS [5] uses queuing network model to estimate capacity and selects paths with sufficient remaining capacity to accommodate the bandwidth demands. A routing method based on minimizing the Lyapunov drift is proposed in [11]. It uses a tuple-based multidimensional conflict graph model to design multipath routing. For the part of improved solution, CSO-AODV [12] improves the cuckoo search algorithm and applies it to route reply. Then the best available path considering QoS can be selected. The QoS guaranteed routing in [13] integrates the strength of PSO and GA. It can find an optimal path which reduces the cost and also satisfies the QoS constraints.

However, existing research does not consider and give relationship between delay and interference. If the delay can be expressed by the simple factor such as the number of interfering nodes, it can be evaluated easily without using proactive probes. Heavy load and congestion at a node can decrease the network performance, so load should be balanced when finding the solution of routing optimization problem. Besides, the solution of routing can influence the network condition in turn. This influence should also be considered when selecting the optimal path.

\section{ROUTING WITH THE OBJECTIVE OF DELAY MINIMIZATION (RODM) DESIGN}

RODM formulates the best route selection as a mathematical optimization problem. As delay is a very important network performance metric nowadays, RODM sets minimizing delay as its objective. Considering interference, SINR, the asymmetry of link and transmission failure probability, the mathematical expression of delay is given. To adapt to typical routing problem in multi-hop WMN, an improved GA which can balance load is proposed to solve the optimization problem.

\section{A. System model}

A network connectivity graph $G=(V, L)$ is used to describe WMN. $V$ is the set of mesh nodes, and $L$ is the set of all links in WMN. Mesh nodes are uniformly deployed in the network, and multiple orthogonal channels are randomly banded with multiple radio interfaces of mesh routers. Several data flows (i.e., $F$ ) are given.

To evaluate the path loss, both free space propagation model and two-ray ground reflection model [14] are used. Then the path loss effect between node $i$ and node $j$ (i.e.,
$\left.P L_{i j}\right)$ can be expressed as

$$
P L_{i j}=\frac{P_{i j}}{P_{T i}}=\left\{\begin{array}{c}
\frac{G_{t} G_{r} \lambda^{2}}{\left(4 \pi d_{i j}\right)^{2}}, 0<d_{i j} \leq d_{0} \\
\frac{G_{t} G_{r} h_{t}^{2} h_{r}^{2}}{\left(d_{i j}\right)^{4}}, d_{0}<d_{i j} \leq r_{T}
\end{array}\right.
$$

where $h_{t}$ and $h_{r}$ are heights of transmitting and receiving antennas, and $\lambda$ is the wavelength. $d_{i j}$ is the distance between node $i$ and $j$, and $r_{T}$ is the transmission range. $G_{t}$ and $G_{r}$ are the gain of transmitting and receiving antennas respectively. $P_{i j}$ is the receiving signal power at destination node $j$ from source node $i . P_{T i}$ is the transmission power of source node $i . d_{0}$ is the critical distance, and $d_{0}=\frac{4 \pi h_{t} h_{r}}{\lambda}$.

The IEEE 802.11 standard [15] is used as the Medium Access Control protocol. The backoff process will start if the wireless channel is contended. Retransmission can be caused if transmission is not successful before the maximum number of retransmission is reached. A packet can be seen as transmitted successfully if the source node can receive the Acknowledgement (ACK) from destination. Thus, the link quality of forward and reverse directions should be considered at the same time.

\section{B. Optimization model}

1) Objective: Minimizing end-to-end delay is set as the objective of RODM, and it is given as

$$
\text { minimize }\left\{\sum_{(i, j) \in L} \sum_{e \in F} X_{i j}^{e} T_{(i, j)}^{e}\left(N_{i}^{e}+1\right)\right\}, \forall e \in F
$$

where $X_{i j}^{e}$ is the binary decision variable of the optimization problem to show whether flow $e$ is via link $(i, j)$. If link $(i, j)$ is chosen and used to transmit packets of data flow $e$, then $X_{i j}^{e}$ is 1 , or $X_{i j}^{e}$ is $0 . T_{(i, j)}^{e}$ is the delay cost by transmitting each packet on link $(i, j)$ for data flow $e . N_{i}^{e}$ is the number of data packets waiting to be transmitted in data flow $e$ at mesh node $i$. This objective is to minimize the end-to-end delay in multi-hop WMN for each data flow.

As the delay to transmit each packet on link $(i, j)$ (i.e., $\left.T_{(i, j)}\right)$ is composed of transmission and backoff time [16], after some simplification, it can be expressed as

$$
\begin{aligned}
& E\left[T_{(i, j)}\right]=\frac{\text { Length }}{B_{(i, j)}}\left[\frac{1-p_{l(i, j)}^{K}}{1-p_{l(i, j)}}\right] \\
& +\frac{C W_{\min }\left[1-\left(2 p_{l(i, j)}\right)^{K+1}\right] \cdot \text { SlotTime }}{2\left(1-2 p_{l(i, j)}\right)}-\frac{\left(1-p_{l(i, j)}^{K}\right) \cdot \text { SlotTime }}{2\left(1-p_{l(i, j)}\right)}
\end{aligned}
$$

where $p_{l(i, j)}$ is the transmission failure probability between nodes $i$ and $j$, and it is based on the transmission failure probability of forward and reverse links. Length is the size of data packet, $K$ is the largest allowed number of retransmission, $B_{(i, j)}$ is the bandwidth of link $(i, j)$. When the number of retransmission is beyond the maximum allowed number $K$, the packet will be dropped. SlotTime is the time of a slot. $C W_{\min }$ is the size of minimum backoff window. Thus the delay is related to the available bandwidth and transmission failure probability.

(1) Available bandwidth 
The available bandwidth is related to the SINR, and the SINR is determined by the receiving power of signal and interference.

According to formula (1), the receiving power of signal from node $i$ to node $j$ (i.e., $P_{i j}$ ) is

$$
P_{i j}=P_{T i} \cdot P L_{i j}=\left\{\begin{array}{c}
P_{T i} \cdot \frac{G_{t} G_{r} \lambda^{2}}{\left(4 \pi d_{i j}\right)^{2}}, 0<d_{i j} \leq d_{0} \\
P_{T i} \cdot \frac{G_{t} G_{r} h_{t}^{2} h_{r}^{2}}{\left(d_{i j}\right)^{4}}, d_{0}<d_{i j} \leq r_{T}
\end{array}\right.
$$

Then the receiving power of interference to link $(i, j)$ (i.e., $\left.I_{i j}\right)$ is

$$
I_{i j}=\sum_{\forall a \in V, a \neq i \quad \sum_{b \in V, b \neq j}} P_{T a} \cdot P L_{a j} \cdot X_{a b} \cdot X_{i j},
$$

where $a$ and $b$ are the source and destination nodes of interfering link $(a, b)$ respectively. The total receiving power of interference is the sum of power of all interfering links.

Then the available bandwidth can be expressed as

$$
\begin{array}{r}
B_{(i, j)}=B_{0} \cdot \log _{2}\left(1+\mathrm{SINR}_{i j}\right)=B_{0} \cdot \log _{2}\left(1+\frac{P_{i j}}{N+I_{i j}}\right), \\
\forall i, j \in V, i \neq j
\end{array}
$$

where $B_{0}$ is the nominal bandwidth, $S I N R_{i j}$ is the SINR of link $(i, j)$ and $N$ is the power of background noise.

(2) Transmission failure probability

When the SINR is lower than threshold, the destination node cannot decode signal successfully and transmission will be failed. A successful transmission needs the source node receiving ACK. Thus the transmission failure probability of the reverse link should also be considered. The total transmission failure probability between nodes $i$ and $j$ is based on the transmission failure probabilities of the forward and reverse links. Based on the relationship between receiving power and distance, the probability density function of receiving signal and interference power can be obtained. Thus the transmission failure probability of forward link $(i, j)$ is

$$
\begin{aligned}
& p_{(i, j)}=p\left(S I N R_{i j}<\beta\right)=p\left(\frac{P_{i j}}{N+\sum_{a \in I_{j}} P_{a j}}<\beta\right) \\
& \approx p\left(P_{i j}<\beta \sum_{a \in I_{j}} P_{a j}\right)=p\left(P_{x}<\beta n P_{a}\right) \\
& =\int_{-\infty}^{+\infty} f_{P_{a j}}\left(P_{a}\right) \cdot p\left(P_{x}<n \beta P_{a} \mid P_{a}\right) d P_{a} \\
& =\int_{-\infty}^{+\infty} f_{P_{a j}}\left(P_{a}\right) \int_{-\infty}^{n \beta P_{a}} f_{P_{i j}}\left(P_{x}\right) d P_{x} d P_{a}
\end{aligned}
$$

where $\beta$ is the threshold of SINR. $I_{j}$ is the set of interfering nodes of node $j . n$ is the size of $I_{j} . f_{P_{i j}}\left(P_{x}\right)$ and $f_{P_{a j}}\left(P_{a}\right)$ are the probability density functions of receiving signal and interference power respectively. In the interference limited network, interference from other transmitters is much larger than the white noise in the receivers, so the background noise can be ignored [17] [18]. Similarly, the transmission failure probability of reverse link $(j, i)$ (i.e., $\left.p_{(j, i)}\right)$ can also be obtained. Then the total transmission failure probability between node $i$ and $j$ (i.e., $p_{l(i, j)}$ ) is

$$
p_{l(i, j)}=1-\left(1-p_{(i, j)}\right) \cdot\left(1-p_{(j, i)}\right)
$$

After some simplification, $p_{l(i, j)}$ can be expressed as

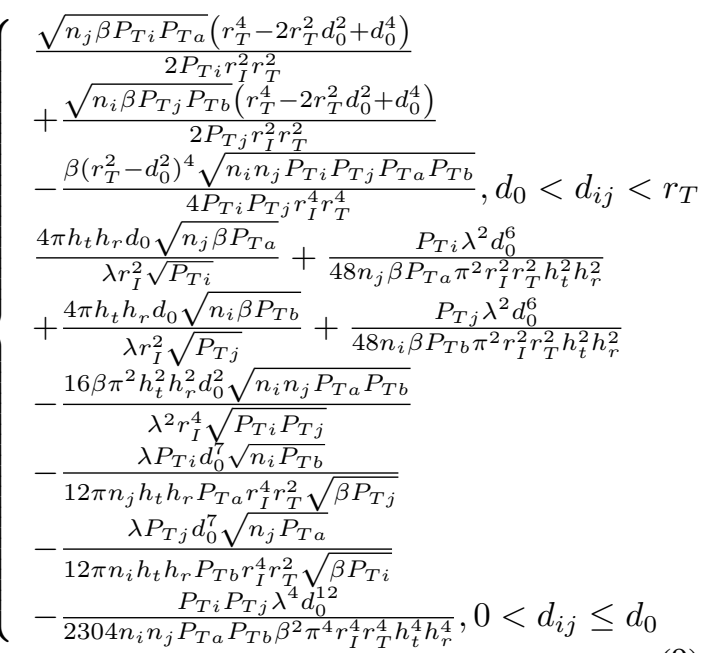

Thus, according to the available bandwidth and transmission failure probability, the expression of delay can be obtained, and the relationship between delay and interference is built. Delay can then be evaluated by the number of interfering nodes in RODM, which is simple and useful. As the solution of path selection can influence the interference condition, RODM checks the number of interfering nodes every time of finding optimal paths in the iterative optimization.

2) Constraints: The source node of each data flow must connect one neighbor node to transmit data packets out:

$$
\sum_{(i, j) \in L} X_{i j}^{e}=1, i \in S, \forall e \in F
$$

where $S$ is the set of source nodes. Similarly, the destination also needs to connect one neighbor node to receive data packets:

$$
\sum_{(i, j) \in L} X_{i j}^{e}=1, j \in D, \forall e \in F
$$

where $D$ is the set of destination nodes. Then the intermediate nodes should guarantee that all the receiving packets can leave through another link:

$$
\sum_{(i, j) \in L} X_{i j}^{e}=\sum_{(j, u) \in L} X_{j u}^{e}, \forall j \in V-\{S, D\}, \forall e \in F
$$

where $u$ is the next hop of node $j$ in data flow $e$.

Besides, the wireless links selected to be used must can exist in the WMN:

$$
c_{i j}^{e} \geq X_{i j}^{e}, \forall(i, j) \in L, \forall e \in F
$$

where $c_{i j}^{e}$ is a binary variable to show whether link $(i, j)$ exists in the network. If the distance between nodes $i$ and $j$ is within the transmission range of each other, then link $(i, j)$ exists and the value of $c_{i j}^{e}$ is 1 . Otherwise, the value of $c_{i j}^{e}$ is 0 .

The optimization model then can be formulated with the objective (2), subject to the constraints (10)-(13). 


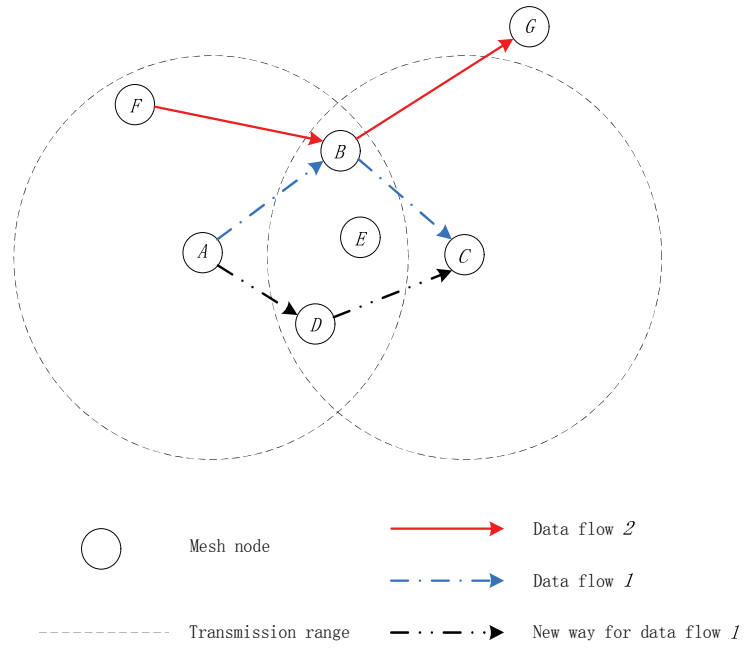

Fig. 2. An example of selecting the replacement node

\section{Solution of optimization model}

Due to the interaction between the solution of routing and the network condition, the formulated optimization problem is difficult to be solved by the conventional optimization algorithms such as the mathematical programming. On the other hand, GA is applicable to the given optimization problem. Further, GA is even regarded as the best choice for finding optimal path [7]. Thus, GA is used to solve the formulated optimization problem in RODM. Some random and valid paths are set as the initial population.

Two main parts of GA are crossover and mutation. In the process of crossover, RODM uses single-point crossover. Two parent chromosomes have a common node, and this common node is the crossover point. The former and latter parts of these two parent chromosomes will be exchanged, and two new chromosomes are produced. In this way, the solution area of RODM can be extended.

In the mutation part, standard GA does mutation randomly without considering the typical routing condition in multihop WMN. Same nodes can be used to transmit multiple data traffic. To balance load effectively, RODM improves the standard GA to avoid using a same node by multiple data flows. When more than one data flows use a same node, the improved GA in RODM will mutate this node to another neighbor, which can also guarantee the transmission requirement. If several neighbors are available, according to the relationship between delay and number of interfering nodes in formulas (3) and (9), the neighbor with the least number of interfering nodes will be selected. A simple example of this method is shown in Fig. 2.

In Fig. 2, we can see that there are two data flows which use a same node $B$. The improved GA in RODM can mutate node $B$ to node $D$ or node $E$ to transmit data flow 1 . In this way, the heavy load and congestion at node $B$ can be effectively reduced. Assume node $D$ has the minimum number of interfering nodes, then node $D$ will be selected to replace node $B$.
TABLE I

SIMULATION PARAMETERS

\begin{tabular}{ll}
\hline Simulation Parameters & Values \\
\hline Simulation time & $100 \mathrm{~s}$ \\
Traffic type & UDP \\
Packet size & 1024 bytes \\
Number of mesh routers & 25 \\
Number of mesh clients & 50 \\
Number of radio interfaces in each router & 3 \\
Number of radio interfaces in each client & 1 \\
Transmission range & $250 \mathrm{~m}$ \\
Interference range & $550 \mathrm{~m}$ \\
Antenna & Omnidirectional \\
\hline
\end{tabular}

\section{Simulation Evaluation}

\section{A. Simulation environment}

Simulation is implemented through NS3 [19], and the simulation area is $1000 \mathrm{~m} \times 1000 \mathrm{~m}$. The packet transmission rate and the length of paths are changed to evaluate the performance of RODM. The detailed simulation parameters are shown in Table I.

\section{B. Performance metrics}

Average packet loss rate, average delay and average network throughput are used to evaluate the network performance.

- Average packet loss rate (i.e., $\mathrm{Pl}$ )

$$
P l=\frac{N_{\text {loss }}}{N_{\text {total }}}=\frac{N_{\text {total }}-N_{\text {received }}}{N_{\text {total }}}
$$

where $N_{\text {loss }}$ is the number of packets which are lost during transmission. $N_{\text {total }}$ is the total number of packets sent by the source. $N_{\text {received }}$ is the number of packets received successfully by the destination.

- Average delay (i.e., $D$ )

$$
D=\frac{\sum_{i=1}^{m} D_{i}}{m}
$$

where $D_{i}$ is the delay cost to transmit packet $i . m$ is the total number of packets received by the destination in success.

- Average network throughput (i.e., $T h$ )

$$
T h=\frac{N_{\text {received }} \times \text { Byte } \times 8}{\left(T_{\text {end }}-T_{\text {start }}\right) \times 1024}
$$

where $N_{\text {received }}$ is the number of packets received by the destination. Byte is the number of bytes contained in one data packet. $T_{\text {end }}$ is the time when the last packet is received successfully. $T_{\text {start }}$ is the time of starting to send the first data packet. The unit of average network throughput is kbps.

\section{Simulation results}

The performance of RODM, search-based routing [7] and routing method with minimized hop count (minHop) is evaluated and compared. The minHop is the most widely used routing method, and it is always used as the benchmark 


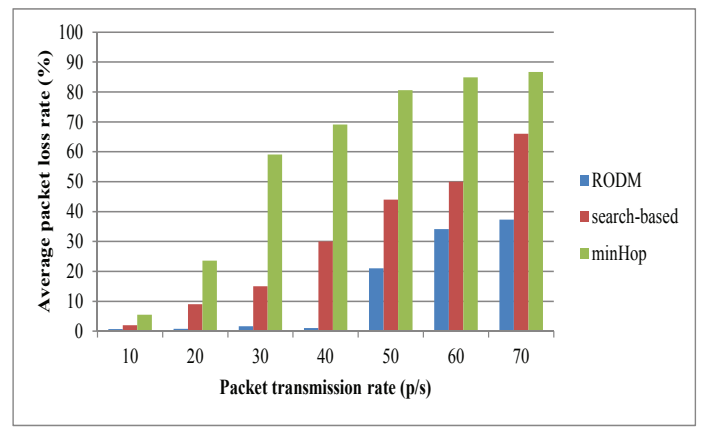

Fig. 3. Average packet loss rate with different packet transmission rate

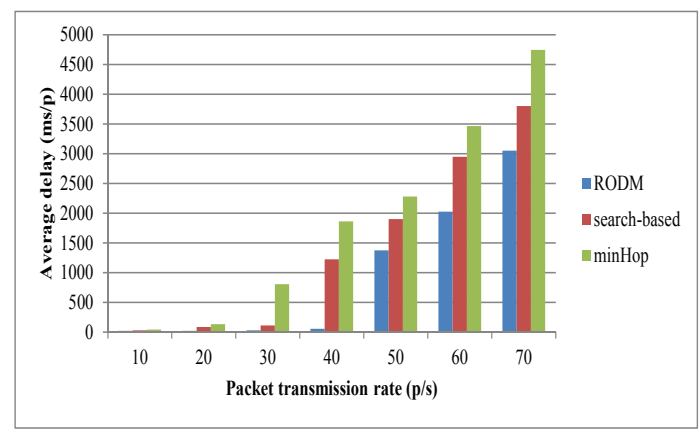

Fig. 4. Average delay with different packet transmission rate

[7] [20]. Besides, the search-based routing proposed in [7] is quite relevant to RODM, and therefore it is also used to compare.

When there are 4 data flows in the network, the simulation results with different packet transmission rate are given in Fig. 3 - Fig. 5.

Fig. 3 - Fig. 5 show RODM can obtain better network performance in terms of average packet loss rate, average delay and network throughput. With the increasing packet transmission rate, the network is more likely to be congested. The minHop only selects path with the least hop count, but neglects network condition. As a result, the path with heavy congestion and bad condition may be selected. Thus, minHop gets the worst performance. The searchbased method considers the expected transmission time on each link, so it can get better performance than minHop.

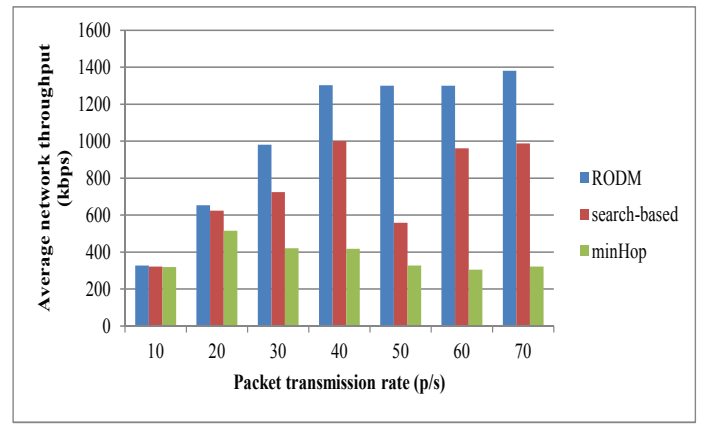

Fig. 5. Average network throughput with different packet transmission rate

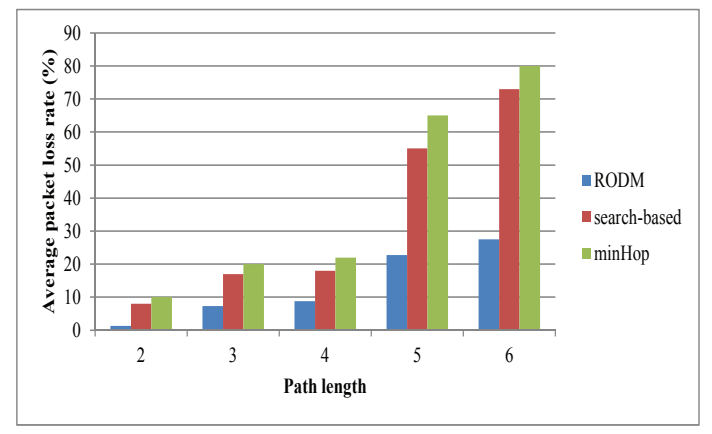

Fig. 6. Average packet loss rate with different path length

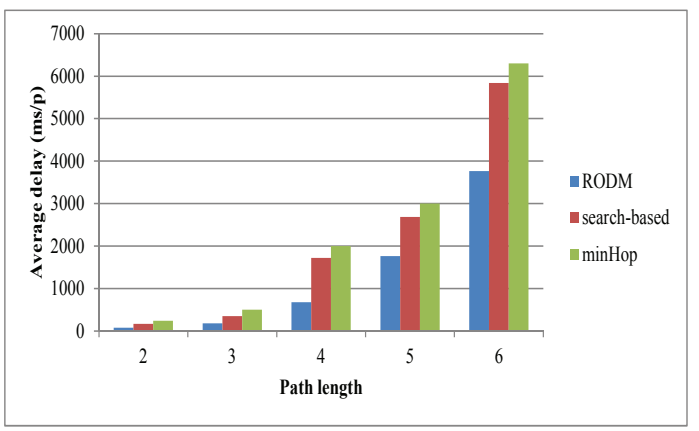

Fig. 7. Average delay with different path length

However, this method neglects the load balance problem, and assumes the values of expected transmission time of all links in the network are known and constant. In fact, these values will be variable when selecting different paths, but the search-based method overlooks this feature, which makes it not very accurate. RODM can effectively avoid using a same congested node, and will find another node with the least number of interfering nodes to replace it. High congestion can then be avoided and heavy load can be balanced in this way. Besides, RODM also considers the dynamic network condition caused by different selected paths when finds routing solution, which leads to choose routes more effectively and accurately. Due to these advantages, RODM performs best in the simulation.

When the number of data flows is 4 , the simulation results with different average path length (that is hop count) are shown in Fig. 6 - Fig. 8.

From Fig. 6 - Fig. 8, we can see that RODM can achieve lower average packet loss rate, lower average delay and higher network throughput than the search-based routing method and minHop. When the path is longer, the probability of interference will become larger. Unlike minHop and search-based method, RODM considers interference condition, and builds the relationship between SINR, transmission failure probability, bandwidth and delay. Then the delay can be evaluated easily and effectively when finding the solution of routing. In addition, when calculating the transmission failure probability, the asymmetry of link is taken into account. Then the more practical transmission failure probability can be obtained. Finally, the paths with 


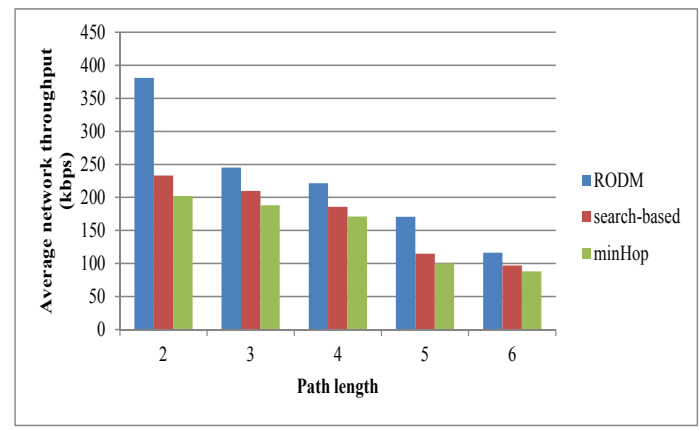

Fig. 8. Average network throughput with different path length

less delay, less interference, large bandwidth and SINR will be selected to service data flows, which brings high performance of the whole network.

\section{CONCLUSION}

Routing is an essential part of network configuration, and it can be formulated as an optimization problem. As more and more services require short delay in rapid development of wireless communication nowadays, RODM proposed in this paper builds an optimization model with the objective of minimizing delay to find optimal paths. Delay is derived by considering interference, SINR, available bandwidth and asymmetrical transmission failure probabilities. The relationship between delay and the number of interfering nodes is then established, which gives a simple way to evaluate delay. When solve the optimization problem, standard GA is improved in RODM to avoid a same node used by multiple data flows. The same node will be replaced by an available neighbor node with the least number of interfering nodes. Load can be balanced effectively in this way. Besides, because the different solutions of routing can influence the network condition such as interference relationship, RODM checks the number of interfering nodes every time when finding optimal paths in the iterative optimization. Simulation results through NS3 show that RODM can find effective paths to gain better network performance.

\section{REFERENCES}

[1] I. F. Akyildiz, X. Wang, and W. Wang, "Wireless mesh networks: a survey," Computer Networks, vol. 47, no. 4, pp. 445-487, Mar. 2005.

[2] E. Di Pascale, I. Macaluso, A. Nag, M. Kelly, and L. Doyle, "The network as a computer: A framework for distributed computing over IoT mesh networks," IEEE Internet of Things Journal, vol. 5, no. 3, pp. 2107-2119, Apr. 2018.

[3] D. Benyamina, A. Hafid, and M. Gendreau, "Wireless mesh networks design-A survey," IEEE Communications surveys \& tutorials, vol. 14, no. 2, pp. 299-310, 2012.

[4] S. Khan, K. K. Loo, N. Mast, and T. Naeem, "SRPM: secure routing protocol for IEEE 802.11 infrastructure based wireless Mesh networks," Journal of Network and Systems Management, vol. 18, no. 2, pp. 190-209, Jun. 2010.

[5] E. Ancillotti, R. Bruno, M. Conti, and A. Pinizzotto, "Load-aware routing in mesh networks: Models, algorithms and experimentation," Computer Communications, vol. 34, no. 8, pp. 948-961, Jun. 2011.

[6] D. Whitley, "A genetic algorithm tutorial," Statistics and computing, vol. 4, no. 2, pp. 65-85, Jun. 1994.
[7] K. Mahmood, B. Nazir, I. A. Khan, and N. Shah, "Search-based routing in wireless mesh network," EURASIP Journal on Wireless Communications and Networking, vol. 36, https://doi.org/10.1186/s13638017-0818-2, Dec. 2017.

[8] V. A. Siris, G. Stamatakis, and E. Tragos, "A simple end-to-end throughput model for 802.11 multi-radio multi-rate wireless mesh networks," IEEE Communications Letters, vol. 15, no. 6, pp. 635-637, Apr. 2011.

[9] C. H. Liu, A. Gkelias, K. K. Leung, "A cross-layer framework of QoS routing and distributed scheduling for mesh networks," in VTC Spring - IEEE Vehicular Technology Conference, pp. 2193-2197, Singapore, May 2008

[10] E. Alotaibi, V. Ramamurthi, M. Batayneh, and B. Mukherjee, "Interference-aware routing for multi-hop wireless mesh networks," Computer Communications, vol. 33, no. 16, pp. 1961-1971, Oct. 2010.

[11] X. Cao, L. Liu, W. Shen, and Y. Cheng, "Distributed scheduling and delay-aware routing in multihop MR-MC wireless networks," IEEE Transactions on Vehicular Technology, vol. 65, no. 8, pp. 6330-6342, Aug. 2016.

[12] V. V. Mandhare, V. R. Thool, and R. R. Manthalkar, "QoS Routing enhancement using metaheuristic approach in mobile ad-hoc network," Computer Networks, vol. 110, pp. 180-191, Dec. 2016.

[13] V. Sarasvathi, N. C. S. N. Iyengar, and S. Saha, "QoS Guaranteed Intelligent Routing Using Hybrid PSO-GA in Wireless Mesh Networks," Cybernetics and Information Technologies, vol. 15, no. 1, pp. 69-83, 2015.

[14] A. U. Chaudhry, R. H. Hafez, O. Aboul-Magd, and S. A. Mahmoud, "Throughput improvement in multi-radio multi-channel 802.11 a-based wireless mesh networks," in Global Telecommunications Conference (GLOBECOM), pp. 1-5, USA, Dec. 2010.

[15] Y. Cheng, X. Ling, W. Song, L. X. Cai, W. Zhuang, and X. Shen, "A cross-layer approach for WLAN voice capacity planning," IEEE Journal on Selected Areas in Communications, vol. 25, no. 4, pp. 678-688, May 2007.

[16] H. Li, Y. Cheng, C. Zhou, and W. Zhuang, "Minimizing end-to-end delay: A novel routing metric for multi-radio wireless mesh networks," in IEEE INFOCOM, pp. 46-54, Rio de Janeiro, Brazil, Apr. 2009.

[17] S. Kandukuri, and S. Boyd, "Optimal power control in interferencelimited fading wireless channels with outage-probability specifications," IEEE transactions on wireless communications, vol. 1, no. 1, pp. 46-55, Jan. 2002.

[18] N. Chakchouk, and B. Hamdaoui, "Traffic and interference aware scheduling for multiradio multichannel wireless mesh networks," IEEE Transactions on Vehicular Technology, vol. 60, no. 2, pp. 555-565, Feb. 2011.

[19] ns-3. http://www.nsnam.org/. Accessed January 9, 2019.

[20] M. Camelo, C. Omana, H. Castro, "QoS routing algorithms based on multi-objective optimization for mesh networks," IEEE Latin America Transactions, vol. 9, no. 5, pp. 875-881, Sept. 2011. 